\title{
Surface-plasmon-enhanced multiple-photon photoemission from Ag and Al films
}

\author{
H. Chen, J. Boneberg, and P. Leiderer \\ Fakultät für Physik, Universität Konstanz, W-7750 Konstanz, Germany \\ (Received 21 August 1992)
}

\begin{abstract}
Photoemission from $\mathrm{Ag}$ and $\mathrm{Al}$ films, mediated by surface plasmons, was studied using a $Q$-switched Nd:YAG laser (where YAG denotes yttrium aluminum garnet) as a light source in the attenuated-totalreflection configuration. Both the intensity dependence of the photoemission yield and the pulse width of the photoemission signal show for metal one-, two-, and three-photon processes for the laser wavelengths $\lambda=355,532$, and $1064 \mathrm{~nm}$, respectively.
\end{abstract}

\section{INTRODUCTION}

Photoemission from metal surfaces is usually a linear process, restricted to incident photon energies above the work function $W$ of the metal. For high incident light intensities, however, nonlinear processes can give rise to photoemission also for photon energies $h v<W .^{1-7}$ The photoelectron yield may be enhanced by several orders of magnitude - compared to the value for the direct photoemission process - when surface plasmons are excited, ${ }^{8}$ which is generally realized in the attenuated-totalreflection configuration. It is believed that in this case the photoemission arises from a coupling of surface plasmons to individual electrons. ${ }^{8}$ Only a few investigations have been reported so far about this surfaceplasmon-mediated photoemission: Early measurements by Bösenberg ${ }^{9}$ and Macek, Otto, and Steinmann ${ }^{10}$ reported electron emission from Cs-coated Ag and Al films, respectively. A quadratic behavior of the photoemission with respect to the incident intensity was measured by Rudolph and Steinmann ${ }^{11}$ using $2.6-\mathrm{eV}$ photons on an Al film, as well as by Chou, Villagrán, and Thompson ${ }^{12}$ using photon energies between 2.3 and $2.6 \mathrm{eV}$ on a $\mathrm{NH}_{3}$ covered Ag film. Two-photon photoemission ${ }^{13}$ was also found by Tsang, Srinivasan-Rao, and Fischer ${ }^{8}$ for an $\mathrm{Ag}$ film using 90-fs laser pulses with a photon energy of 1.9 $\mathrm{eV}$. For $\mathrm{Cu}, \mathrm{Al}$, and $\mathrm{Au}$ these measurements yielded a three-photon process when the peak intensity of the laser pulse was used for the analysis. Surprisingly, however, the temporal profiles of the photocurrent, as compared to the incident laser intensity, did not display the narrowing one would expect for higher-order processes.

Motivated by this discrepancy we have undertaken a systematic study of surface-plasmon-enhanced photoemission from metal films on a nanosecond time scale and at photon energies of $1.17,2.33$, and $3.49 \mathrm{eV}$, where processes of different order should be relevant.

\section{EXPERIMENT}

The metal films studied were Ag and Al films, which were thermally evaporated in a separate vacuum chamber onto BK7 glass prisms at room temperature at a base pressure of $10^{-6}$ mbar. The optimum film thicknesses for plasmon excitation as calculated from the Fresnel coefficients (50 nm for $\mathrm{Ag}$ and $19 \mathrm{~nm}$ for $\mathrm{Al}$ film) were used. ${ }^{14}$ The work functions of thin $\mathrm{Al}$ and $\mathrm{Ag}$ films reported in Ref. 8 were 3.9 and $3.65 \mathrm{eV}$, smaller than those of the bulk crystal, which were $4.5 \mathrm{eV}$ for $\mathrm{Ag}$ and $4.2 \mathrm{eV}$ for A1. For our films the work functions of the films have been determined to be below $3.5 \mathrm{eV}$, since the photoelectron yield measured with a cw argon laser (350-360 nm) exhibited a linear intensity dependence.

Figure 1 shows the schematic setup of the experiment. The prism was mounted in a vacuum chamber at a pressure below $10^{-7}$ mbar. The measurements were performed in the Kretschmann configuration, where a $p$ polarized laser beam irradiates the metal film from the glass side at the surface-plasmon resonance angle. ${ }^{14}$ The light source was a $Q$-switched Nd:YAG (yttrium aluminum garnet) laser, which could be frequency doubled and tripled, with pulse durations of 10,7 , and $5 \mathrm{~ns}$ at the wavelengths 1064,532 , and $355 \mathrm{~nm}$. In order to reduce space-charge effects an extraction voltage of $1 \mathrm{kV}$ was applied between the metal film $M$ and the anode $A$, which was fixed $3 \mathrm{~mm}$ in front of the metal film. The films were checked by optical microscopy after irradiation by the laser beam to ensure that the surface had not been damaged. The ns time-resolved photoemission and the laser pulse shape, measured by a pin diode with rise time $<1$ ns, were recorded with a HP 54111 digital storage oscilloscope $(1 \mathrm{GSa} / \mathrm{s})$. In addition, the laser energy was monitored by a laser power meter. The photoemission signals were obtained at the angle of the maximum electron yield, which differs slightly form the plasmon resonance angle. 8,15

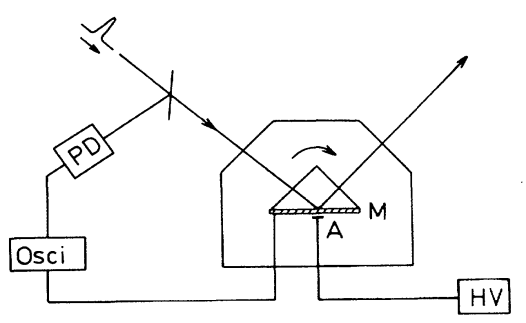

FIG. 1. Schematic arrangement of the experiment. PD, photodiode; Osci, oscilloscope; $\mathrm{HV}$, high voltage; $M$, metal film; $A$, copper anode. 


\section{RESULTS AND DISCUSSION}

\section{A. Intensity dependence of the photoemission yield}

For all three wavelengths the behavior of the electron emission yield was studied as a function of the input laser intensity. Results are shown in Figs. 2-4, where the peak intensities of the photocurrent are plotted versus the peak intensity of the incident laser pulse on a double logarithmic scale.

At the shortest wavelength, $355 \mathrm{~nm}(3.49 \mathrm{eV})$, a linear dependence of the photoelectron yield is found for both metal films, as shown in Fig. 2 for the case of Ag. This is as expected for one-photon photoemission and agrees with the results of the cw laser measurement in the same wavelength range mentioned above.

A distinct change in the power dependence is observed when the photon energy is reduced to $2.33 \mathrm{eV}(532 \mathrm{~nm}$, frequency-doubled Nd:YAG laser pulse), which is too low for a one-photon process to occur. The data now closely follow a quadratic behavior, indicated by the straight line with a slope of 2 in Fig. 3. Hence one is dealing with a second-order process in this case, where two photons (or plasmons) are involved. The quantum efficiency reaches $10^{-9}$ at a laser intensity of $6 \mathrm{MW} / \mathrm{cm}^{2}$.

For the lowest photon energy studied here, $1.16 \mathrm{eV}$ (1064 $\mathrm{nm}$ ), even a second-order process is not sufficient to create photoelectrons, but rather the next-higher-order process is required. In fact the log-log plot of the photoelectron yield for this wavelength (Fig. 4) shows a slope of three, indicative of a three-photon process.

\section{B. Time-resolved photoemission profile}

In this section we discuss the time-resolved photoemission profiles $J_{e}(t)$ in comparison with the temporal profile of the incident laser pulse $I_{l}(t)$, which also ought to reflect the order of the process responsible for photoemission. Yet, as already pointed out in the Introduction, the narrowing of the pulse profile expected for higher-order processes had not been found in recent investigations on a femtosecond scale. ${ }^{8}$

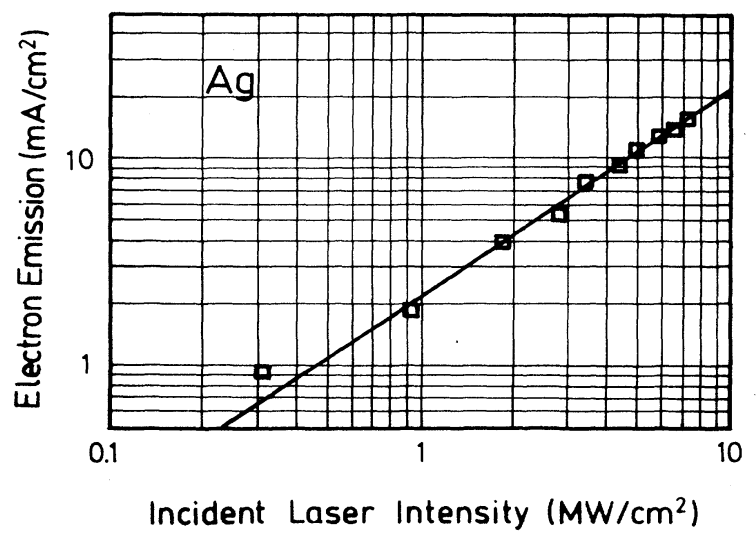

FIG. 2. Photoemission yield of an $\mathrm{Ag}$ film as a function of the incident laser intensity at a wavelength of $355 \mathrm{~nm}$. The straight line represents a linear dependence.

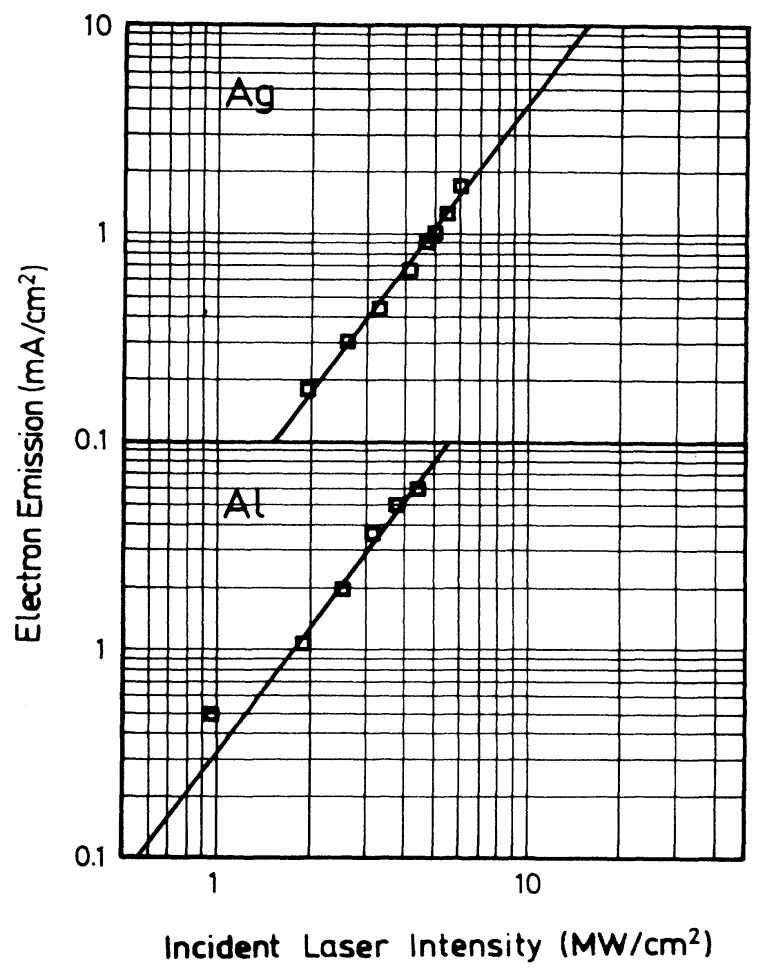

FIG. 3. Photoemission yield of $\mathrm{Ag}$ and $\mathrm{Al}$ films as a function of the incident laser intensity at a wavelength of $532 \mathrm{~nm}$. The straight line represents a quadratic dependence.

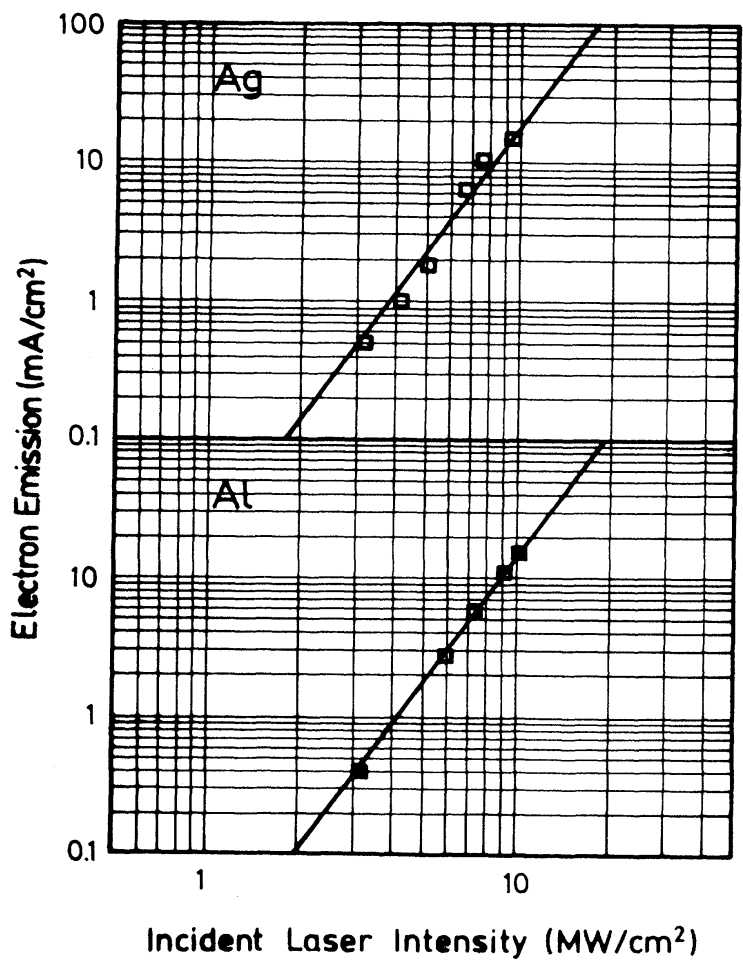

FIG. 4. Photoemission yield of $\mathrm{Ag}$ and $\mathrm{Al}$ films as a function of the incident laser intensity at a wavelength of $1064 \mathrm{~nm}$. The straight line represents a cubic dependence. 
For the $n$ th-order process, where $J_{l} \propto I_{e}^{n}$, the halfwidths of the photoemission pulse ought to be reduced by a factor of $n^{1 / 2}$ compared to the incoming pulse, provided the laser pulse has a Gaussian shape. Since in the present study the temporal profile of the laser pulse was not exactly Gaussian, this relation for the half-width, which was used in Ref. 8, does not strictly apply here. For this reason we directly compare in the following the $n$th power of the measured laser profile with the normalized photoemission signals.

For a linear process, as was deduced from Fig. 2 for the highest photon energy of $3.49 \mathrm{eV}$, the electron emission signal $J_{e}(t)$ should agree in its shape with the laser pulse profile $I_{l}(t)$. Figure 5(a) demonstrates that this is confirmed within our experimental resolution.

Figure 5(b) refers to a photon energy of $2.33 \mathrm{eV}$, where according to the data presented in the previous section we expect a second-order process. Indeed, the temporal profile of the photoemission current agrees very well with the squared laser pulse shape. Similarly, in Fig. 5(c) close agreement between the photoemission signal and the third power of the laser pulse is observed, in line with a three-photon process expected to be relevant in this case.

In order to visualize the narrowing of the photoelectron signal as compared to the incident light intensity, we have plotted in Figs. 5(b) and 5(c) also the time profile of the laser pulse (dashed line).

In summary, the multiphoton processes in photoemission, which manifest themselves in the functional dependence of the photocurrent on the incident laser intensity, were studied on a nanosecond time scale. Both for $\mathrm{Ag}$ and Al films our data for the peak values of the photocurrent yield suggest first-, second-, and third-order processes at laser wavelengths $\lambda=355,532$, and $1064 \mathrm{~nm}$. A comparison of the temporal profiles of the electron pulse and the incident laser pulse is completely consistent with this interpretation. A discrepancy between the analysis of peak intensity and half-width of the pulses, as reported by Tsang, Srinivasan-Rao, and Fischer $^{8}$ for a similar study on surface-plasmon-enhanced photoemission on a femtosecond time scale, was not found here. This difference is probably due to the lifetime of the surface plasmons, ${ }^{16,17}$ which is much shorter than the width of our laser pulses, but is comparable to the pulse duration in the measurement of Tsang, Srinivasan-Rao, and Fisch-

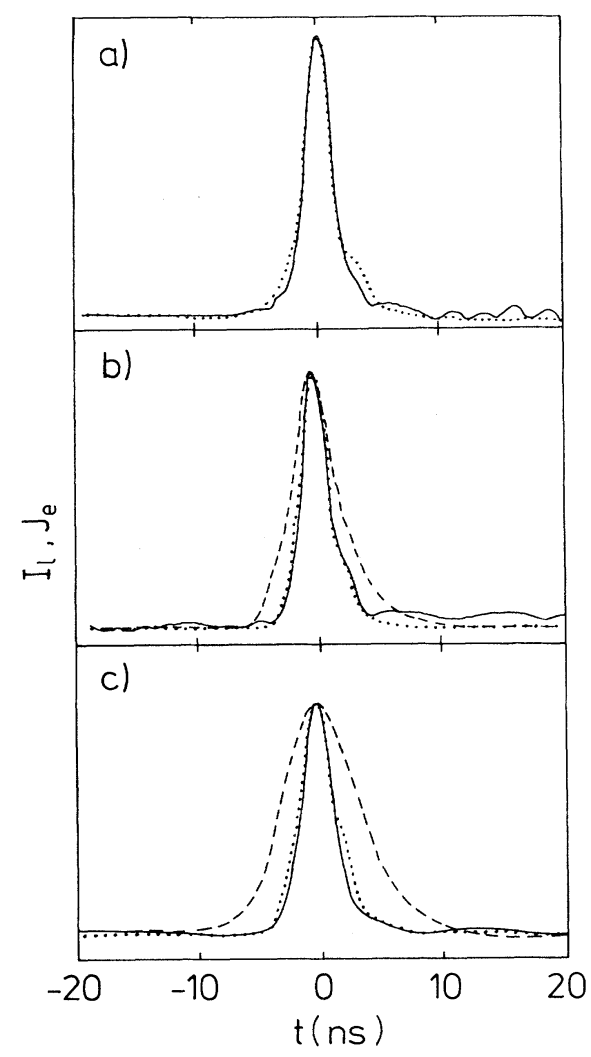

FIG. 5. Time-resolved photoemission signal $J_{e}$ (full curve) in comparison with the incident laser pulse. (a) $\lambda=355 \mathrm{~nm}$; dotted line: normalized laser pulse $I_{l}$; (b) $\lambda=532 \mathrm{~nm}$; dotted line: $\left(I_{l}\right)^{2}$; dashed line $I_{l}$; (c) $\lambda=1064 \mathrm{~nm}$; dotted line: $\left(I_{l}\right)^{3}$ dashed line: $I_{l}$.

er. ${ }^{8}$ With the surface plasmons being responsible for the photoemission process, their lifetime poses a lower limit on the pulse width of the photoelectrons.

\section{ACKNOWLEDGMENTS}

We appreciate many fruitful discussions with S. Herminghaus. This work was supported by Chinese Academic Society (H.C.) and Zentrum II für Energieforschung at Konstanz University (J.B.).
${ }^{1}$ J. G. Endriz, Phys. Rev. B 7, 3464 (1973).

${ }^{2}$ J. H. Bechtel, W. Lee Smith, and N. Bloembergen, Phys. Rev. B 5, 4557 (1977).

${ }^{3}$ R. Yen, J. Liu, and N. Bloembergen, Opt. Commun. 35, 277 (1980).

${ }^{4}$ R. R. Freeman et al., Phys. Rev. Lett. 59, 1092 (1987).

${ }^{5}$ E. Yablonovitch, Phys. Rev. Lett. 60, 795 (1988).

${ }^{6}$ S. D. Moustaizis et al., Appl. Phys. Lett. 58, 194 (1991).

${ }^{7}$ P. G. Strupp, P. C. Stair, and E. Weitz, J. Appl. Phys. 69, 3472 (1991).

${ }^{8}$ T. Tsang, T. Srinivasan-Rao, and J. Fischer, Phys. Rev. B 43, 8870 (1991).

${ }^{9}$ J. Bösenberg, Phys. Lett. 37A, 439 (1971).

${ }^{10} \mathrm{Ch}$. Macek, A. Otto, and W. steinmann, Phys. Status Solidi B
51, K59 (1972).

${ }^{11}$ H. W. Rudolf and W. Steinmann, Phys. Lett. 61A, 471 (1977).

${ }^{12}$ H.-T. Chou, J. C. Villagrán, and J. C. Thompson, Phys. Rev. B 44, 3359 (1991).

${ }^{13}$ Although photoemission is mediated by surface plasmons in the experiments to be discussed here, the term two- and three-photon photoemission is used in the literature for the higher-order processes.

${ }^{14}$ H. Raether, Surface Plasmons (Springer, Berlin, 1986).

${ }^{15}$ G. Hincelin, Phys. Rev. B 24, 787 (1981).

${ }^{16}$ R. H. M. Groeneveld, R. Sprik, and A. Lagendijk, Phys. Rev. Lett. 64, 784 (1990).

${ }^{17}$ N. Kroo et al., Europhys. Lett. 15, 289 (1991). 